\title{
Frauen in Roben - auf dem Weg nach oben
}

Eva Schübel

Vizepräsidentin

des djb/Gleichstel-

lungsbeauftragte

des Generalbundes-

anwalts

\section{Für Richterinnen und Richter, Staatsanwältinnen und \\ Staatsanwälte, die minderjährige Kinder oder \\ pflegebedürftige Angehörige betreuen, sind an deren \\ besondere Lebenssituation angepasste Bewährungs- \\ und Aufstiegsmöglichkeiten zu schaffen.}

Kontakt zu den Justizministerien und den dortigen Gleichstellungsbeauftragten.

Chancengerechtigkeit muss Berufsverbänden ein besonderes Anliegen sein. Sie spielt freilich beim Deutschen Richterbund (DRB) anders als beim djb eine nur marginale Rolle, obwohl unter seinen rund 15.500 Mitgliedern viele Richterinnen und Staatsanwältinnen sind. Wir wollen dies in einem neuen Netzwerk von djb-Frauen, die auch dem DRB angehören, diskutieren.

\section{A) Frauen in die Roten Roben: Ziel erreicht?}

Vor welchem Hintergrund ist der Erfolg bei der Bundesrichterwahl am 22. Mai 2014 zu sehen? Ist die djb-Initiative „Frauen in die Roten Roben“ damit erledigt? Anlass für deren Gründung war die Wahl im März 2011, als nur 15 Prozent Richterinnen vorgeschlagen und 16,7 Prozent gewählt worden waren. Wir empfanden es als Skandal, dass unter den 23 Kandidierenden für den Bundesgerichtshof (BGH) nur drei Richterinnen waren und die Vorschlagsliste für das Bundesverwaltungsgericht (BVerwG) 16 Männer und nur eine Frau aufwies. Wir haben daraufhin geeignete Kandidatinnen gesucht und die zuständigen Gleichstellungsbeauftragten der Länderjustiz und bei den Bundesgerichten in die Initiative eingebunden. Das hat sich ausgezahlt. Für die Richterwahl 2012 wurde schon ein Drittel Frauen vorgeschlagen. Nun ist der weibliche Anteil auf 47 Prozent gestiegen. Einen Ausreißer bildet wieder das BVerwG mit zehn Bewerbern und nur fünf Bewerberinnen; immerhin sind zwei Frauen und zwei Männer gewählt worden.

In diesem Ergebnis dürfte sich das in der neuen Legislaturperiode weiblichere Wahlgremium bemerkbar gemacht haben. Es war eine unserer Forderungen, die bei der Neubesetzung der Richterwahlausschüsse vor allem durch die SPD erfüllt worden ist: die beiden Wahlgremien (für das Bundesverfassungsgericht und die obersten Bundesgerichte) sind nun auf Seiten der Abgeordneten mit 50 Prozent bzw. mit fast 50 Prozent Frauen besetzt. Aber auch unsere vielfältigen Kontakte zu den Mitgliedern des Richterwahlausschusses dürfte zu dem sensationellen Wahlergebnis von 55 Prozent Frauen beigetragen haben. Noch im Zeitraum zwischen 2008 und 2012 gingen 78 Prozent der Stellen für den BGH an Männer (vgl. Brückner, djbZ 2013, 191 [193]). Der beträchtliche Anstieg von 16,7 Prozent über 30 Prozent und 44 Prozent im letzten Jahr auf nun 55 Prozent gewählte Frauen lässt auf ein grundlegendes Umdenken im Wahlgremium hoffen. Darauf verlassen sollten wir uns noch nicht: wir müssen den öffentlichen Druck auf die Richterwahlgremien erhalten. Ein Wahlergebnis, das qualifizierte Kandidatinnen unberücksichtigt lässt, ist nicht zu verantworten.

Vor diesem Hintergrund darf auch die Vorschlagsquote von 50 Prozent nicht mehr sinken. Wer sich mit dem Verfahren der Bundesrichterwahl befasst, stößt sehr schnell auf die Frage, wie jemand auf die Vorschlagslisten kommt. Ob die Bestenauslese gilt, ist zumindest unklar. Denn es gibt weder Anforderungsprofile für die einzelnen Bundesgerichte noch ein gemeinsames Grundprofil für die Verwendung an den obersten Gerichtshöfen. Die Stellen werden in der Regel weder bekannt gemacht noch die Gleichstellungsbeauftragten an der Auswahl der Kandidierenden beteiligt. Lediglich in der Berliner Arbeitsgerichtsbarkeit wird seit Jahren ein Interessenbekundungsverfahren in Bezug auf das Bundesarbeitsgericht (BAG) durchgeführt.

Wir fordern deshalb seit Beginn der Initiative mehr Transparenz. Für die diesjährige Wahl konnte die Gleichstellungsbeauftragte der Justiz Schleswig- 
Holstein erreichen, dass erstmals die Richterinnen und Richter über das Wahlverfahren und die Stellensituation bei den Bundesgerichten informiert sowie ihnen die Möglichkeit einer Bewerbung gegeben wurde (Bekanntmachung des MJKE vom 22.1.2014). Meine Anfrage bei den an den Bundesrichterwahlen beteiligten Ministerien, insbesondere den 16 Justizministerien, hat bislang in Niedersachsen ein Umdenken bewirkt, wo es künftig ein Interessenbekundungsverfahren vor jeder Bundesrichterwahl geben wird. Brandenburg und Sachsen-Anhalt werden immerhin eine Einführung prüfen. Zumindest die Beteiligung der Gleichstellungsbeauftragten gibt es schon in Bayern, RheinlandPfalz, dem Saarland und künftig in Sachsen.

Eine Chance, vorgeschlagen und gewählt zu werden, sollen auch Kandidierende erhalten, die aus familiären Gründen teilzeitbeschäftigt sind. Denn Akademikerinnen bekommen ihre Kinder häufig erst mit Mitte 30 und haben daher im üblichen Wahlalter von circa 42 bis 50 Jahren betreuungsintensiven Nachwuchs. Der Bundesjustizminister hat diese Forderung des Arbeitskreises der Gleichstellungsbeauftragten bei den Bundesgerichten, dem Bundesverfassungsgericht (BVerfG) und dem Generalbundesanwalt aufgenommen und an die Mitglieder des Richterwahlausschusses herangetragen. Allerdings ist an den obersten Bundesgerichten das Festhalten an (männlich geprägten) Traditionen noch groß, so dass es längere Zeit dauern dürfte, bis eine Teilzeittätigkeit bei Bundesrichterinnen und -richtern, aber auch schon bei den wissenschaftlichen Mitarbeitenden, selbstverständlich sein wird.

Der eben genannte Arbeitskreis fordert auch ein Teilnahmerecht der Gleichstellungsbeauftragten der Bundesgerichte an den Sitzungen und Beratungen der Präsidialräte, die die persönliche und fachliche Eignung der Kandidierenden bewerten. Dieses Recht für die Gleichstellungsbeauftragten, das die Schwerbehindertenvertreter bereits haben, ist nun im Entwurf eines Gesetzes für die gleichberechtigte Teilhabe von Frauen und Männern an Führungspositionen in der Privatwirtschaft und im Öffentlichen Dienst enthalten. Das Problem des Zugangs zu den Sitzungen der örtlich zuständigen Räte stellt sich ebenso in den Ländern und wird von den Gleichstellungsbeauftragten der Justizministerien angegangen.

\section{B) Frauen in schwarzen Roben: Best Practice-Modelle zur Förderung der Gleichstellung}

Bei meinem Berufsstart 1985 gab es unter mehr als 40 Richtern und Staatsanwälten im Landgerichtsbezirk Hof nur zwei Frauen. Die mit mir und nach mir in den Justizdienst strebenden jungen Kolleginnen haben bald gemerkt, wie mühsam sich Familie und Beruf, geschweige denn Familie und Karriere, vereinbaren ließen. Dass Richterinnen und Staatsanwältinnen mit Kindern beim beruflichen Aufstieg benachteiligt werden, ist seit der vom Bundesjustizministerium in Auftrag gegebenen Studie „Frauen in der Justiz - eine empirische Analyse der Berufssituation, Karriereverläufe und Karrierechancen von Richterinnen, Staatsanwältinnen und Rechtspflegerinnen “von 1992 bekannt (veröffentlicht in der Reihe Rechtstatsachenforschung, Bundesanzeiger 1993). Das im Grundgesetz seit 1994 verankerte Gebot an staatliche Stellen, die Gleichberechtigung von Frauen und Männern tatsächlich durchzusetzen und bestehende Nachteile zu beseitigen, hat an den Aufstiegshindernissen für Richterinnen und Staatsanwältinnen wenig geändert.

20 Jahre später sind Kinder weiterhin das Karrierehemmnis schlechthin. Zu diesem Ergebnis gelangte die Forschungsstudie „Frauen in Führungspositionen der Justiz - Eine Untersuchung der Bedingungen von Frauenkarrieren in den Justizbehörden in Nordrhein-Westfalen“ aus dem Jahr 2011 (Zusammenfassung durch Schultz, DRiZ 2012, 264). Im Wesentlichen verantwortlich dafür sind erhebliche strukturelle Mängel in der Beförderungspraxis der betroffenen nordrhein-westfälischen Justiz. Solche althergebrachten Strukturen, die Richterinnen und Staatsanwältinnen mit Familienpflichten benachteiligen, sind in der Länderjustiz generell zu finden. Teilzeitbeschäftigte sind - wegen der nur dort gegebenen zeitlichen Flexibilität - hauptsächlich an den Amtsgerichten mit den geringeren Aufstiegschancen tätig. Da familiäre Aufgaben die zeitliche und räumliche Flexibilität einschränken, ist für sie ein Durchlaufen der Regelerprobung oft schwer möglich oder sogar unmöglich. Sie erhalten kaum karriererelevante Positionen, wie zum Beispiel Sonderverwendungen in der Gerichtsverwaltung, für die regelmäßig volle Verfügbarkeit und ständige Anwesenheit im Büro erwartet werden. Vorgesetzte, für die immer noch der zeitlich umfassend verfügbare Richter oder Staatsanwalt das Richtmaß bildet, beurteilen Teilzeitbeschäftigte tendenziell schlechter und sprechen zum Beispiel in NordrheinWestfalen laut Studie die männlichen Kollegen früher auf eine Bewerbung an, was sich auf das Bewerbungsverhalten und die Beförderungschancen auswirkt.

Die Ergebnisse der Studie von 2011 dürfen deshalb nicht nur in Nordrhein-Westfalen Beachtung finden. Zwar gibt es in einzelnen Ländern bereits positive Ansätze für mehr Chancengleichheit auf dem Weg in Führungspositionen und erfolgreiche Maßnahmen, die als Best Practice-Modelle dienen könnten. Veränderungen sind aber bundesweit u.a. im Beurteilungswesen, bei Erprobung und Sonderverwendungen erforderlich. Ich wollte deshalb wissen, wie sich die Länderjustiz zu diesen Fragen verhält. Ich habe im März 2014, wie oben schon erwähnt, die an den Bundesrichterwahlen beteiligten Ministerien, neben den Justizministerien auch das Bayerische Innen-, Finanz- und Arbeitsministerium sowie die für den Bereich Arbeit zuständige Berliner Senatorin, angeschrieben und um Auskunft u.a. zu folgenden Fragen gebeten:

Werden offene Stellen ausgeschrieben, insbesondere auch Sonderverwendungen? Wird die Gleichstellungsbeauftragte bei deren Besetzung beteiligt? Welche Führungspositionen und Sonderfunktionen können in Teilzeit ausgeübt werden? Wie ist im Beurteilungssystem sichergestellt, dass Verzerrungen in der Beurteilung (z.B. bei Teilzeitkräften, nach Elternzeit u.ä.) sichtbar werden? Gibt es in den Flächenstaaten gleichwertige wohnortnahe Erprobungsalternativen?

Geantwortet haben bisher 16 Ministerien (von 20). Es fehlen Bremen, Hamburg, Mecklenburg-Vorpommern und SchleswigHolstein. Ich stelle im Folgenden von den Ländern genannte Maßnahmen dar, die auf gleichberechtigte und uneingeschränkte 
Karrieremöglichkeiten abzielen. Da nicht alle Ministerien zu allen Fragen detailliert Auskunft gegeben haben, beansprucht die Aufzählung der angegebenen Bundesländer keine Vollständigkeit.

1. Beurteilung: Ein Notenspiegel ist das wirksamste Instrument zu prüfen, ob einzelne Gruppen (z. B. Frauen gegenüber Männern, Teilzeitbeschäftigte gegenüber Vollzeitkräften, Richter/innen am AG gegenüber Richter/innen am LG) schlechter beurteilt werden, was erfahrungsgemäß nicht selten vorkommt. Die nach Frauen/ Männern, Teilzeit/Vollzeit etc. aufgeschlüsselten Beurteilungsergebnisse werden statistisch ausgewertet und auf Diskrepanzen untersucht (Bayern), ggfs. sind Gegenmaßnahmen zu treffen.

Bei Elternzeit sind Beurteilungslücken in der Leistungsentwicklung zu vermeiden. Zwei Instrumente helfen: Die Anlaßbeurteilung vor Antritt einer Elternzeit (Sachsen-Anhalt ab 3 Monate, Rheinland-Pfalz und Saarland ab 1 Jahr Dauer) und die fiktive Nachzeichnung der Leistungsentwicklung beurlaubter Kräfte (Nordrhein-Westfalen).

2. Sonderverwendungen: Es wird umfassend über sämtliche Sonderverwendungen informiert (Personalentwicklungskonzept Baden-Württemberg), die Stellen werden ausgeschrieben (Berlin), z.B. per Rundmail (Arbeitsgerichtsbarkeit Berlin) oder Sharepoint (Rheinland-Pfalz), und die Gleichstellungsbeauftragte an der Auswahlentscheidung beteiligt (Berlin). Alle Sonderverwendungen einschließlich Führungs-Trainee-Programme (Brandenburg) können in Teilzeit ausgeübt werden (BadenWürttemberg, Saarland; nur grundsätzlich möglich in Berlin, Nordrhein-Westfalen, Rheinland-Pfalz).

3. Erprobung: Sofern nicht generell auf eine Erprobung verzichtet wird (Bayern), ist Teilzeitbeschäftigten der Eignungsnachweis in Teilzeit und ohne Verlängerung der Dauer möglich (z.B. Brandenburg, Hessen, Sachsen-Anhalt). Die Zahl der TeilzeitStellen entspricht dem Bedarf (strebt Nordrhein-Westfalen an). Zeitliche Flexibilität verschaffen individuell gestaltete Arbeitszeitmodelle (Rheinland-Pfalz mit einem Frauenanteil von knapp 45 Prozent bei der Erprobung im Jahr 2012) und die Ausstattung der Erprobungsarbeitsplätze mit Notebooks (Niedersachsen). Ein häuslicher Arbeitsplatz reduziert die Anwesenheitstage (Nordrhein-Westfalen), wobei es einen Anspruch auf Heimarbeit geben sollte. Urlaub ist während der Erprobung in den Schulferien möglich (Rheinland-Pfalz).

Eine Heimerprobung am bisherigen Dienstort können Beschäftigte machen, denen aus wichtigen familiären Gründen die Regelerprobung nicht möglich ist (Niedersachsen; SachsenAnhalt mit der Einschränkung, dass eine Erprobung beim oberen Landesgericht und einem anderen Gericht oder einer anderen Staatsanwaltschaft unzumutbar ist).

Die Kriterien und Mindestvoraussetzungen für eine Erprobungsabordnung werden bekannt gemacht (Baden-Württemberg). Über offene Erprobungsstellen wird informiert (Brandenburg). Die Gleichstellungsbeauftragte ist in die Besetzung der Erprobungsstellen eingebunden (Hessen).

4. Beförderung: Beförderungsämter mit Führungs- bzw. Leitungsfunktion können in Teilzeit wahrgenommen werden (Baden-Württemberg, Berlin, Hessen, Niedersachsen, Rheinland-Pfalz; in Sachsen grundsätzlich möglich; in Bayern und im Saarland mit Ausnahme der Leitung von Gerichten und Staatsanwaltschaften). Bei Führungspositionen gilt der Grundsatz, dass Teilzeitbeschäftigung - von absoluten Ausnahmen abgesehen - organisierbar ist, u.a. mit häuslichem Arbeitsplatz (Bayerisches Staatsministerium für Arbeit und Soziales, Familie und Integration - BayStMAS).

Im Fall der Unterrepräsentanz von Frauen wird bei gleicher fachlicher Befähigung und Eignung eine Frau ausgewählt (Baden-Württemberg).

Diese Übersicht zeigt, dass es wirksame Maßnahmen für mehr Familienfreundlichkeit, Geschlechtergerechtigkeit und die dazu unerlässliche Frauenförderung gibt. Dies erfordert keine Teilnahme an der Auditierung „berufundfamilie“ (BayStMAS 2005, JM BW 2014, BMJV 2009), diese hilft aber überkommene Strukturen und Denkweisen zu ändern. Eine rechtliche Verpflichtung besteht indes, diskriminierende Vorschriften und eine Verwaltungspraxis, die sich am typischen Karriereverlauf eines Mannes orientiert, zu ändern. Der Grundsatz der Gleichbehandlung verbietet nach der Rechtsprechung des Bundesverfassungsgerichts nicht nur eine Benachteiligung derjenigen, die aus familiären Gründen Auszeiten nehmen müssen und/oder keinen vollen Dienstposten bewältigen können, sondern er gebietet, Personalmaßnahmen an Art. 3 II 2 des Grundgesetzes ausgerichtet anzupassen (BVerfG, NJW 2014, 843). In der Praxis heißt das: Für Richterinnen und Richter, Staatsanwältinnen und Staatsanwälte, die minderjährige Kinder oder pflegebedürftige Angehörige betreuen, sind an deren besondere Lebenssituation angepasste Bewährungs- und Aufstiegsmöglichkeiten zu schaffen, wie zum Beispiel reduzierte Präsenzpflicht, Teilzeittätigkeit in allen Sonderverwendungen und Führungspositionen, wohnortnahe Erprobung. Was bereits erfolgreich in dem einen oder anderen Bundesland praktiziert wird, gilt es nun gebündelt in der gesamten Länderjustiz einzuführen.

Probleme bei der Vereinbarkeit von Familie und Karriere können nicht mehr als individuelle Angelegenheit von Eltern, vor allem von Müttern, angesehen werden, die außerhalb der Zuständigkeit der Personalverantwortlichen liegen. Der Föderalismus ist ein Grund für Vielfalt, gibt aber nicht die Rechtfertigung, Richterinnen und Staatsanwältinnen mit Kindern beim beruflichen Aufstieg weiterhin zu diskriminieren.

\section{C) Berufliche Netzwerke fruchtbar machen}

Nicht erst seit der Initiative „Frauen in die Roten Roben“ hat der djb die Richterinnen und Staatsanwältinnen im Blick. Sie machen immerhin einen Anteil von circa 15 Prozent unserer Mitglieder aus. Vor allem viele der weiblichen Führungskräfte der Justiz sehen die Arbeit des djb als notwendig und sehr positiv an. Der djb hat jedoch nicht den Organisationsgrad des Bundes der Richterinnen und Richter, Staatsanwältinnen und Staatsanwälte (DRB) erreicht, dessen Mitglied ich seit fast 30 Jahren bin. 1985 waren in Hof über 90 Prozent der Kolleginnen und Kollegen Mitglied im Bayerischen Richterverein. Wie ich oben ausführlich dargelegt habe, hat sich an den massiven Karrierehindernissen für Richterinnen und Staatsanwältinnen mit Familienaufgaben seit der Studie „Frauen in der Justiz“ 
von 1992 leider wenig geändert. Familie und Beruf sind vereinbar geworden, nicht aber Familie und Karriere. Dies wirft die Frage auf, was der DRB in den mehr als 20 Jahren gemacht hat. Wäre es nicht auch seine Aufgabe gewesen, auf die Beseitigung der Hindernisse hinzuwirken? Der DRB, ein männlich geprägter Verband, hat die Benachteiligung der Kolleginnen beim beruflichen Aufstieg im Wesentlichen ausgeblendet. Die althergebrachten Beförderungsstrukturen und das nicht seltene Vorurteil von Personalverantwortlichen, Mütter hätten kein Interesse am beruflichen Fortkommen, haben den Kollegen Konkurrentinnen vom Hals gehalten. Der Richterbund mag einwenden, die betroffenen Frauen hätten sich doch im Verband in ihrem Sinne engagieren können. Theoretisch ja, praktisch hatten und haben sie aber in der Regel keinen Partner zuhause, der ihnen den Rücken für Verbandsaktivitäten neben dem Beruf und der Familie frei hält. Die Mitarbeit im DRB ist zweifelsohne karriereförderlich. Wenn eine Frau mittut, wird in der Regel etwas aus ihr und das war es dann in Sachen Frauenförderung.

Der Vorwurf fehlenden Engagements hat allerdings einen wahren Kern: Frauen unterschätzen den Nutzen von beruflichen Netzwerken für ihr Fortkommen (Brückner, djbZ 2013, 191). Leistung und Befähigung sind eine Grundvoraussetzung für Erfolg, sie reichen aber meist nicht, weil Menschen mit eigenen Interessen darüber entscheiden, wer befördert wird. Daher ist es wichtig, Schaltstellen zu erkennen und zu nutzen, die Einfluss auf die eigene Karriere haben. Dazu zählen nicht nur Sonderverwendungen als Präsidialrichter/in und Pressesprecher/ in, sondern auch als Gleichstellungsbeauftragte und ein Engagement in Berufsverbänden. Die örtlichen Verbände des DRB verfügen meist dank ihrer hervorragenden Vernetzung in die Gerichts- und Behördenleitungen über einen sehr guten Einblick in und Einfluss auf die oft verschlungenen Wege, wer wann welche Karriere macht. Kontakte u.a. zu den Führungsspitzen der Justiz können über den djb geknüpft werden, der zudem Frauen bei der Besetzung von Top-Positionen ins Gespräch bringt. Schließlich sollten Richterinnen und Staatsanwältinnen das Amt der Gleichstellungsbeauftragten nicht unterschätzen und für ihre Belange nutzen. Denn es verschafft umfassende Insider-Kenntnisse und gibt das Recht, selbst initiativ zu werden (Schübel, djbz 2013, 86).

Wir müssen keine neuen Wege suchen, sondern uns die vorhandenen Pfade erschließen - frei nach dem Spruch von Simone de Beauvoir, den der Interministerielle Arbeitskreis der Gleichstellungsbeauftragten im Bund propagiert: „Frauen, die nichts fordern, werden beim Wort genommen. Sie bekommen nichts. “

\section{European Women Shareholders Demand Gender Equality: Erstes Projekttreffen in Brüssel}

Das djb-Projekt „European Women Shareholders Demand Gender Equality " hat im April 2014 von der Europäischen Kommission neben 16 weiteren europäischen Projekten den Zuschlag für eine Förderung nach dem PROGRESS-Programm erhalten. Es wird kofinanziert vom Bundesministerium für Familie, Senioren, Frauen und Jugend (BMFSFJ), dem Ministerium für Justiz und Gleichstellung des Landes Sachsen-Anhalt und der Finnischen Handelskammer. Am 16. Mai 2014 war offizieller Projektbeginn.

Am 19. Juli war es endlich soweit: Das djb-Projektteam, nationale Koordinatorinnen und Vertreterinnen der Projektpartner trafen sich erstmals im Rahmen des neuen djb-Projekts „European Women Shareholders Demand Gender Equality“. Ort des

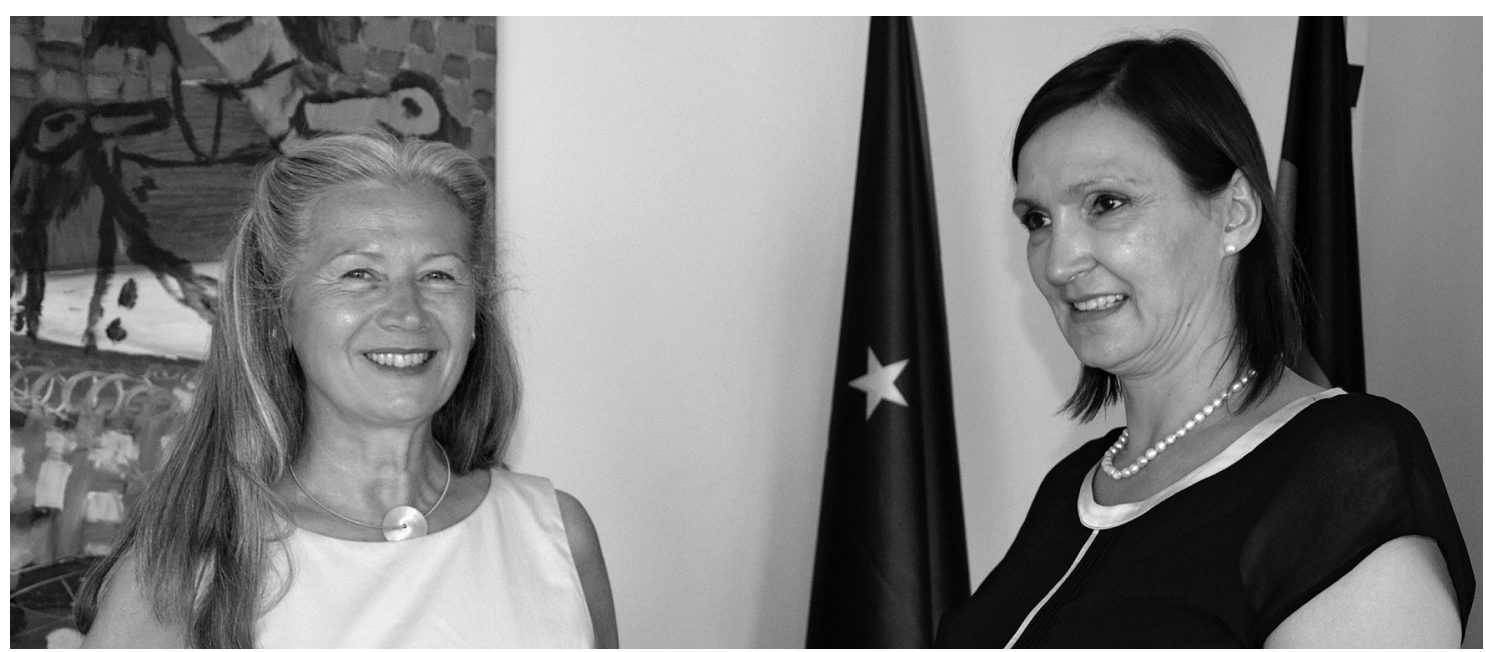

$\Delta$ Ramona Pisal, djb-Präsidentin, und Prof. Dr. Angela Kolb, Ministerin für Justiz und Gleichstellung des Landes Sachsen-Anhalt (Foto: djb)

Miruna Bucurescu, Linda Walczak Projektmitarbeiterinnen, Deutscher Juristinnenbund e.V., Berlin 